whose members fought more often than they worked together. These experiences strengthened my resolve to build my practice the way I wanted it to be. They also reminded me that my patients are people and my interactions with them are more than just tasks to be completed before the end of the day.

My exposure to numerous preceptors was influential on how I viewed not only my personal practice but also pharmacy practice as a whole. They prepared me to be a leader, regardless of my work environment or position:

Leadership ... is being confident in your abilities, knowledge and skills to shape your practice and the practice of the profession. It is about ... being proactive in your approach to patient care ... [and] setting the practice expectations that will have the best impact on patients.-April monthly reflection

As a whole, a residency program is best described by the cliché of the roller coaster ride: you're thrown forward into new practice areas at breakneck speeds, twisted and pulled in every direction with the multiple projects, discussions, presentations, and forms that must be completed, on top of patient care. You're flipped upside down when you realize that processes that worked for you as a student aren't good enough in real-life practice, and life flashes before your eyes while you have very little sense of what is going on in the world around you. Yet, like every good amusement park ride, no matter how terrifying it is, the residency year always ends too quickly and you realize after it's over that you just want to hop back on. Was my year challenging? Yes. But was it worth it? More than words can say.

I've now been in my workplace for 7 months and have seen the positive effect that my residency training has had on me. Naturally, I still experienced the culture shock of working in a new area, and the learning curve has been steep. But I knew my residency training had been worth it when, on the second day of my clinical training, I felt completely at ease on the ward and on rounds despite being new to the service area.

Would I have had such an easy transition without a residency? I don't think so. It would have taken me much longer to find my professional identity without the exposure I had during the residency year. I'm very grateful for the residency experience and for all of the preceptors who gave up their time to coach and guide me. All I can say now is, "Change: Bring it on!"

Ashley Walus, BSCPharm, ACPR

Children's Hospital, Health Sciences Centre

Winnipeg, Manitoba

Competing interests: Ashley Walus is the Manitoba representative on the CSHP 2015 Branch Champions subcommittee.

\section{Optimal Warfarin Management Can Be Achieved in All Practice Settings}

We read with interest the Point Counterpoint column in the September-October 2012 issue of the Canadian Journal of Hospital Pharmacy. Kertland ${ }^{1}$ and Tejani ${ }^{2}$ highlighted many important issues in their debate about whether new oral anticoagulants should replace warfarin for prophylaxis of thromboembolism in Canadians with atrial fibrillation.
We would like to clarify one point regarding the review by the Canadian Agency for Drugs and Technologies in Health (CADTH) on the optimal use of warfarin. CADTH did recommend a well-coordinated, structured approach to warfarin management, ${ }^{3}$ but it did not recommend that care be "dedicated to anticoagulation therapy", as stated by Kertland.

The best available evidence is unclear as to whether specialized anticoagulation clinics result in improved outcomes for patients. What does matter is a structured approach, including a clear plan for patient follow-up, use of a validated dosing tool, ongoing patient education, and involvement of caregivers and other health professionals. This type of care can take place in a specialized anticoagulation clinic, a family doctor's or specialist's office, or other care settings.

CADTH continues to focus on prevention of stroke among patients with atrial fibrillation. A systematic review and network meta-analysis, comparing the new oral anticoagulants with warfarin, was completed in April 2012. ${ }^{4}$ The best available evidence to date suggests that the benefit of the new oral anticoagulants is small, their long-term safety is unknown, and the new drugs are more expensive even when warfarin monitoring is taken into account.

Expert recommendations based on the review were made in June 2012, with warfarin being recommended as first-line therapy for patients with nonvalvular atrial fibrillation. ${ }^{5}$

An extension of this review, which will include antiplatelet agents such as acetylsalicylic acid and clopidogrel, will be available in spring 2013. All CADTH reports are freely available at the organization's website (www.cadth.ca/anticoagulants), which readers may visit for additional information.

\section{References}

1. Kertland H. Should direct thrombin inhibitors replace warfarin for prophylaxis of thromboembolism in Canadians with atrial fibrillation? The "pro" side. Can J Hosp Pharm. 2012;65(5):401-3.

2. Tejani AM. Should direct thrombin inhibitors replace warfarin for prophylaxis of thromboembolism in Canadians with atrial fibrillation? The "con" side. Can J Hosp Pharm. 2012;65(5):403-5.

3. Recommendations for optimal warfarin management for prevention of thromboembolic events in patients with atrial fibrillation. Optimal Use Report, Volume 1, Issue 2c. Ottawa (ON): Canadian Agency for Drugs and Technologies in Health; 2011 Nov [cited 2013 Jan 24]. Available from: www.cadth.ca/media/pdf/OP0508_warfarin_rec-report_e.pdf

4. Wells G, Coyle D, Cameron C, Steiner S, Coyle K, Kelly S, et al. Safety, effectiveness, and cost-effectiveness of new oral anticoagulants compared with warfarin in preventing stroke and other cardiovascular events in patients with atrial fibrillation. Ottawa (ON): Canadian Collaborative for Drug Safety, Effectiveness and Network Meta-Analysis; 2012 Apr [cited 2013 Feb 12]. Available from: www.cadth.ca/media/pdf/NOAC_Therapeutic_Review_final_report.pdf

5. CADTH therapeutic review recommendations: New oral anticoagulants for the prevention of thromboembolic events in patients with atrial fibrillation. Ottawa (ON): Canadian Agency for Drugs and Technologies in Health; 2012 Jun [cited 2013 Feb 12]. Available from: www.cadth.ca/media/pdf/tr0002_New-OralAnticoagulants_rec_e.pdf

Sarah Jennings, BSC, BScPhm, RPh, PharmD

Janice Mann, BSc(Hon Bio and Psych), MD

Knowledge Exchange Officers

Canadian Agency for Drugs and Technologies in Health

Ottawa, Ontario

Competing interests: None declared. 